\title{
Flipped Teaching: una metodología en construcción...
}

Anna Vidal Melóa, Vicente D. Estruch Fuster ${ }^{\mathrm{b}}$, Francisco J. Boigues Planes ${ }^{\mathrm{c}}$, Romina del Rey Tormos ${ }^{\mathrm{d}}$, Jesús Alba ${ }^{\mathrm{e}}$, Bernardino Roig Sala ${ }^{\mathrm{f}}$, Constantino Torregrosa ${ }^{\mathrm{g}}$

aavidal@mat.upv.es, bvdestruc@mat.upv.es, cfraboipl@mat.upv.es, droderey@doctor.upv.es, ejesalba@fis.upv.es, fbroig@mat.upv.es, , ${ }^{\mathrm{g}} \mathrm{ctorregr} @$ fis.upv.es

Grup d'Innovació Educativa i Recerca en Màteries Cientifiques (GIERMAC)

Campus de Gandia de la Universitat Politècnica de València, C. Paranimf, n 1. 46730-Grau de Gandia (Valencia). Teléfono: 962849333. Fax: 962849309.

\section{Abstract}

The UPV promotes the application of new teaching methods, such as the Flipped Teaching or Flipped Classrom, which consists, broadly speaking, of doing at home what is supposed to be done in class (theoretical explanation) and doing in class what is commonly done at home (problem solving).

This approach involves having more time during face-to-face classes for problem solving, practical work, teamwork and so on, thus favouring the application of active methodologies. GIERMAC (Grup d'Innovació Educativa i Recerca en Matèries Cientifiques) takes part in the UPV PIMEs 2015-2016 notification, with PIME A04- "Study on the application of Flipped Teaching in Mathematics and Physics subjects". This paper summarizes the work done so far: planning and selection of contents, selection of resources and the making of Flip programming cards, detailing how the methodology is developed in the study of a specific topic.

Keywords: Flipped Teaching, inverse lecture, active methodologies, Mathematics, Physics.

\section{Resumen}

La UPV potencia la aplicación de nuevas metodologías docentes, como es el caso del Flipped Teaching o clase inversa, que consiste, a grandes rasgos, en hacer en casa lo que tradicionalmente se hace en el aula (la exposición teórica) y realizar en el aula lo que comúnmente se hace en casa (resolución de problemas). Este planteamiento supone disponer de más tiempo en las clases presenciales para la resolución de problemas, realización de prácticas, trabajo en equipo,... favoreciéndose la aplicación de metodologías activas. El Grup d'Innovació Educativa i Recerca en Matèries Científiques (GIERMAC) participa en la convocatoria PIMEs 2015-2016 de la UPV, con el PIME A04"Estudio sobre la aplicación del Flip Teaching en asignaturas de Matemáticas y Física". En este trabajo se resume la labor realizada hasta el momento: planificación y selección de contenidos, selección de recursos, y confección de 
una colección de fichas Flip que detallan la forma en que se desarrolla la metodología en el estudio de un tema concreto.

Palabras clave: Flipped Teaching, Flipped Classrom, metodologías activas, Matemáticas, Física.

\section{Introducción}

Aunque en este trabajo se utiliza la expresión Flipped Teaching, también es habitual la utilización de términos como Flip Teaching, Flipped Classrom o "clase al revés" (Bergamnn y Sams, 2105), o también aula invertida, Flipped Teaching o Flipped Learning.

El Flipped Teaching es un enfoque o modelo pedagógico cuya idea central es "dar la vuelta" al método de enseñanza común o modelo tradicional. A grandes rasgos supone hacer en casa lo que tradicionalmente se hace en el aula (la exposición teórica) y realizar en el aula lo que normalmente se hace en casa (resolución de problemas), aunque esta inversión supone mucho más, tal y como se evidenciará en el trabajo que se presenta.

El término aula invertida, o Inverted Classroom, fue acuñado por un grupo de profesores de economía en la Universidad de Miami (Ohio) y tiene sus raíces en el método del caso utilizado en las escuelas de negocios, derecho y humanidades, donde se pedía a los estudiantes que fuera de clase prepararan una lectura para poder, posteriormente, desarrollar una discusión en clase (Lage, Platt y Treglia, 2000). Posteriormente, en 2007, Jonathan Bergmann y Aaron Sams, dos profesores de química en Woodland Park High School, Colorado, empezaron a grabar en soporte video los contenidos de sus asignaturas y a subirlos a la red.De esta forma subsanaban el problema de la falta de asistencia a clase de algunos alumnos con actividades diversas lejos del centro (Bergamnn y Sams, 2105). Aunque no fueron pioneros en la utilización de vídeos tutoriales, fueron de los primeros en defender esta herramienta como instrumento de enseñanza. Posteriormente, exigieron a sus estudiantes ver un vídeo como tarea en casa, antes de la clase presencial, tomando notas sobre lo aprendido. Así, al no dedicar ese tiempo en clase para escuchar las explicaciones del profesor, disponían de más tiempo presencial, en clase y en laboratorios, para poner en práctica los conocimientos adquiridos, resolver dudas, realizar proyectos o experimentos, aumentando de esta forma la interacción profesor-alumno, y ofreciendo una educación más personalizada. De esta forma invirtieron las actividades con respecto al modelo tradicional, naciendo su "clase al revés".

El modelo actual de educación, es un reflejo de la época en la que ésta se diseñó, en la Revolución industrial, y de ahí que los estudiantes sean educados como una especie de línea de producción: se les pide que se sienten en filas muy ordenadas, que escuchen lo que un experto expone sobre un tema y que lo recuerden ante un examen. La enseñanza tradicional es como el café para todos: presentar tantos contenidos como se pueda en el tiempo de clase y esperar a que lleguen a tantos alumnos como sea posible, (Bergamnn y Sams, 2015, p. 19 
y 20). En la "clase al revés" se rebaja el protagonismo del docente para hacer más énfasis en el del alumno y su aprendizaje.

Entre las ventajas que tiene este nuevo enfoque destacan las siguientes:

- Respecto a la utilización de vídeos didácticos:

- Se habla el mismo idioma que los estudiantes, muy acostumbrados a las tecnologías interactivas y habituados a utilizar vídeos tutoriales de internet para preparar exámenes.

- Ayuda a los alumnos "muy ocupados" o con problemas.

- Permite al profesor pausar y recapitular: no todos los estudiantes tienen el mismo ritmo de aprendizaje, aspecto éste que no se tiene en cuenta en una clase tradicional.

- Respecto a las actividades en la clase presencial:

- Permite ofrecer una retroalimentación inmediata.

- Incrementa la interacción alumno/profesor.

- Permite que los profesores conozcan más y mejor a sus alumnos y que se atienda mejor a los alumnos con problemas.

- Con la realización de trabajos en grupo o colaborativos se mejora la interacción alumno/alumno.

En el vídeo correspondiente a la Figura 1, (Flipping Physics, 2015), se compara, de forma amena y divertida una clase tradicional con una invertida.

Bergmann y Sams (2015) también comprobaron que sus estudiantes aprendían más y sus calificaciones mejoraban. Otros estudios en el ámbito universitario (Talbert, 2012), demuestran la eficacia del Flipped Teaching, tanto a nivel de resultados en el número de aprobados como en la adquisición de determinadas competencias (capacidad de adquirir conocimientos técnicos por su propia cuenta, capacidad de autocorrección, alto nivel de trabajo a pesar de su falta de base inicial,...) en diversas asignaturas y universidades: Biología (Moravec, Williams, Aguilar-Roca y O'Dowd, 2010), Diseño de Software (Gannod, Burge y Helmick, 2008), Álgebra Lineal (Talbert, 2014) e Introducción a la informática Científica (Talbert, 2012).

En España podemos encontrar numerosas páginas web que tratan esta metodología. Entre ellas vale la pena destacar dos, (Figura 2: Javier Touron. Talento-Educación-Tecnología Touron y The flipped classroom). 


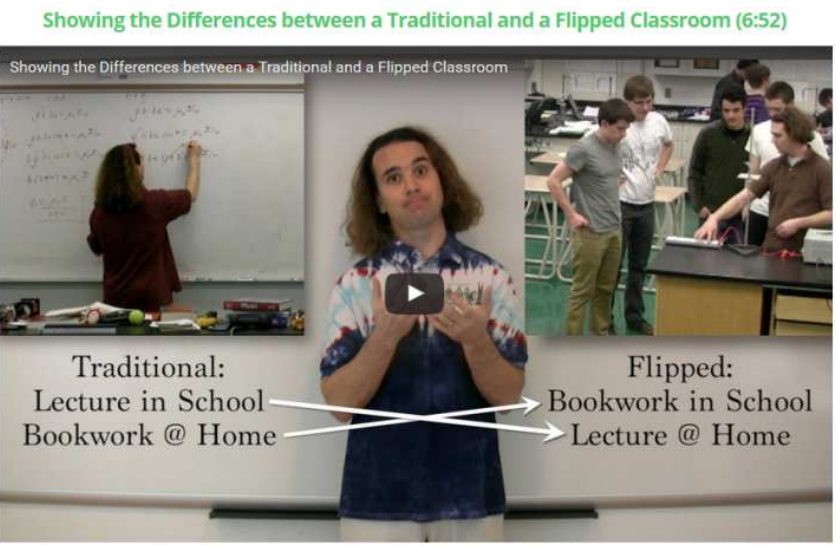

Fig. 1 Diferencias entre una clase tradicional y una clase Flip

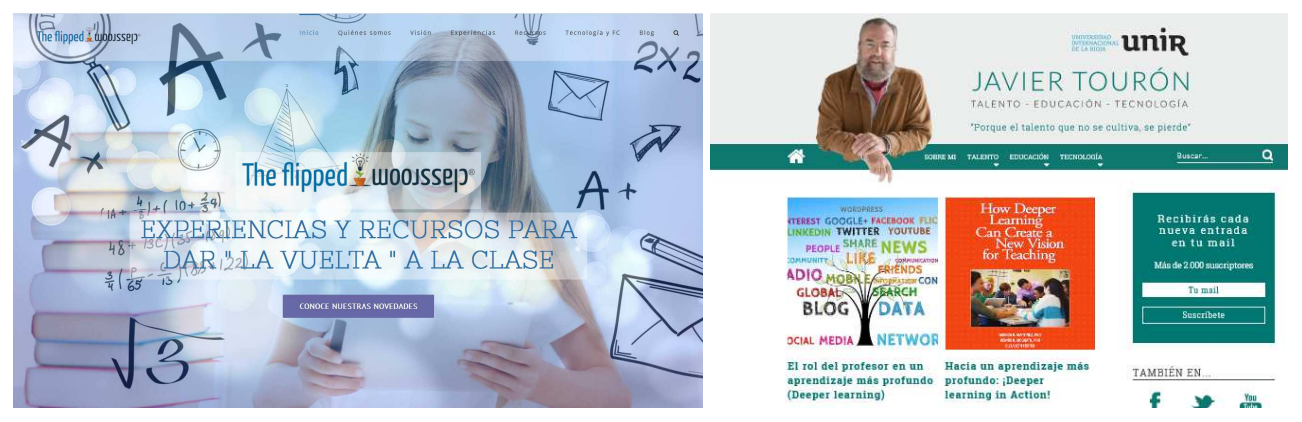

Fig. 2 Portadas principales de las páginas web The flipped classroom y Javier Touron

Entre la gran cantidad de información que contienen, existe un amplio repertorio de infografías, también disponibles en Pinterest, (Figura 3). A pesar de las ventajas descritas respecto a la utilización de vídeos, la segunda infografía de la Figura 3 detalla otras alternativas que pueden utilizarse para que los estudiantes aprendan lo básico, sobre un contenido concreto, fuera del aula.

Pero lo más relevante en el Flipped Teaching no es el uso de vídeos, sino las actividades que los alumnos pueden hacer en el aula. Actualmente, en el ámbito universitario todavía existe bastante confusión entre los docentes, principalmente por desconocimiento del enfoque Flip, que muchos reducen a tener que grabar vídeos. El aula invertida es mucho más, de hecho supone "un enfoque integral para incrementar el compromiso y la implicación del alumno en la enseñanza" (López Moreno, 2015). El Flip no son sólo vídeos online, ni consiste en reemplazar al docente por vídeos.Tampoco se trata de hacer un curso online. Lo interesante e importante es fomentar la relación profesor-alumno con el desarrollo de actividades para las clases presenciales. No es un método para que el estudiante estudie a su antojo o trabaje sólo. El docente debe planificar y estructurar de forma adecuada el aprendizaje del estudiante (Tourón, Santiago y Díez, 2014). 

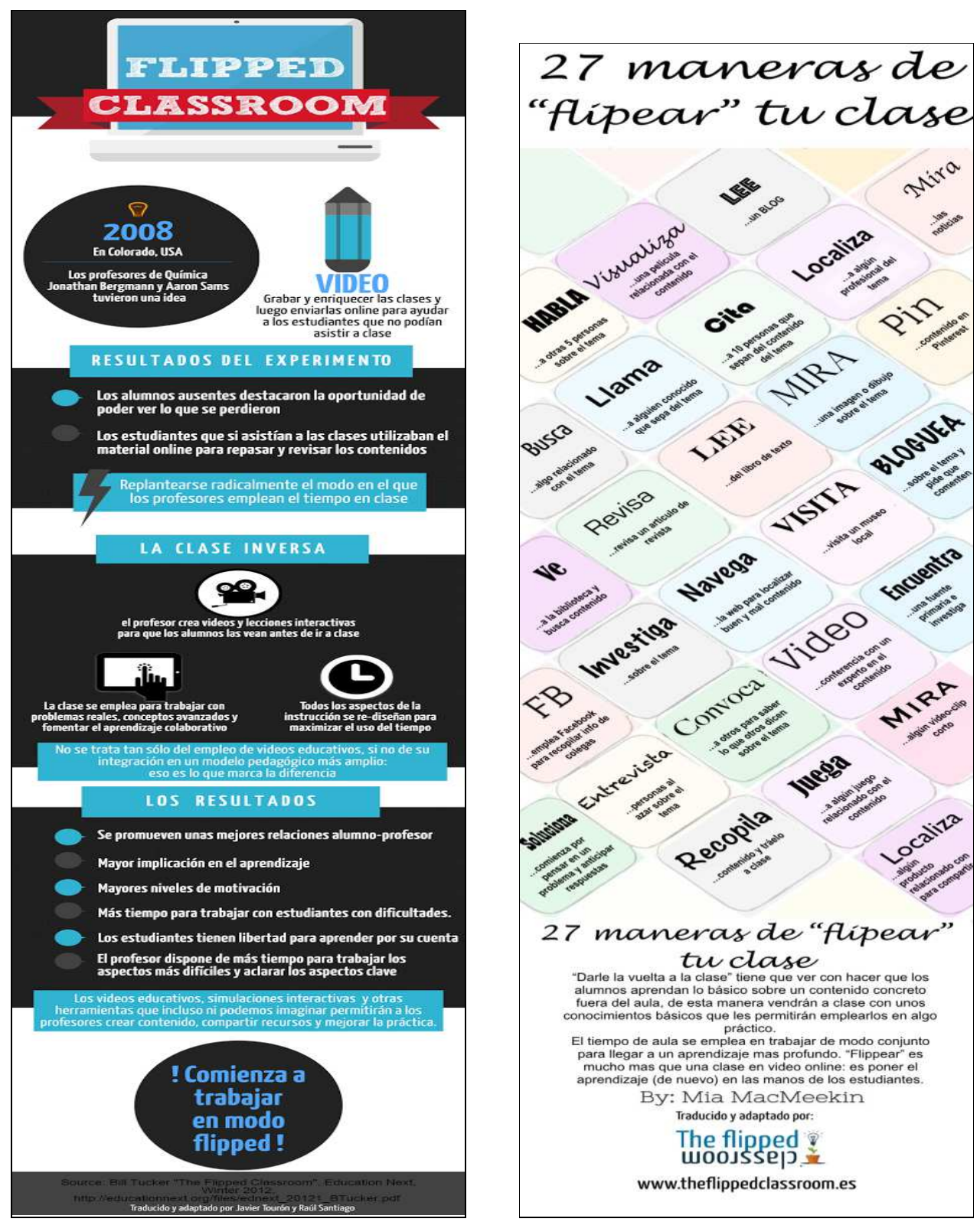

Fig. 3. Primera infografía, de Bill Tucker "The Flipped Classroom", Education Next. Winter 2012. http://educationnext.org/files/ednext 20121 BTucker.pdf. Traducida y adaptado por Javier Tourón y Raúl Santiago. Segunda infografía de Mia MacMeekin, traducida y adaptada por The Flipped Classroom.

La clase inversa supone cambiar la estructura tradicional del aprendizaje en la que el docente suele dedicar mucho tiempo a la explicación de los temas en el aula. Los estudiantes no necesitan al profesor en el aula cuando éste expone los contenidos; sino más bien en la resolución de problemas que realizan, por lo general, fuera de la clase, y por cuenta propia. Por lo tanto, parece razonable una inversión de la clase tradicional, de forma que los estudiantes adquieran la información básica a través de vídeos, lecturas, y otras fuentes, trabajando fuera de clase, dejando la clase presencial para trabajos más activos dinámicos y

(cc) BY-NC-ND 2016, Universitat Politècnica de València

Congreso In-Red (2016) 
participativos, a través de problemas, prácticas o trabajando en equipo. De esta forma se favorece la aplicación de otras metodologías activas como el aprendizaje basado en problemas, en proyectos y otras dinámicas colaborativas. Asi, el docente puede atender a los estudiantes con mayor eficacia, dedicar más tiempo a resolver dudas, a guiar a los estudiantes en las aplicaciones prácticas de la teoría, a observar cómo realizan el trabajo y a guiar la en la solución de los problemas que se encuentran.

Es bastante frecuente que los trabajos en grupo se realicen, totalmente o en parte, en horas no presenciales, pudiendo aparecer problemas: incompatibilidad de horario; falta de compromiso por parte de algún miembro; desigualdad de carga en el trabajo realizado, etc. Realizando estos trabajos en horas presenciales, estos problemas pueden evitarse, al menos en parte, a la vez que el profesor puede observar la adquisición de determinadas competencias generales. En relación con este aspecto, posteriormente se analizará el cambio realizado en las prácticas de una asignatura concreta (Matemáticas 2) para minimizar este tipo de problemas.

No obstante, no basta pues con invertir el orden de presentación de los recursos y actividades; también se ha de plasmar la inversión en la programación, en las actividades que se propongan y, cómo no, en la evaluación. En Hughes (2012) se detalla cómo diseñar diferentes estrategias a tener en cuenta para "dar la vuelta" al aula universitaria y en Touron, Santiago y Díez, (2014), algunas experiencias didácticas.

Sin embargo, para que el proceso de aprendizaje Flip tenga éxito es de suma importancia que el estudiante también se comprometa con su aprendizaje y tome parte activa en el mismo.

\section{Objetivos}

Durante el curso 2014-2015, se desarrolló una experiencia piloto de aplicación de la metodología Flipped Teaching en varias Escuelas de la UPV y el Instituto de Ciencias de la Educación (ICE) de la UPV ha estado impartiendo cursos relacionados con esta metodología. Esto ha motivado al GIERMAC a participar en el PIME A04- "Estudio sobre la aplicación del Flip Teaching en asignaturas de Matemáticas y Física", dentro de la convocatoria PIMEs 2015-2016 de la UPV, y en el que, inicialmente, las asignaturas involucradas, del Campus de Gandia de la UPV, eran:

- Matemáticas 2: asignatura anual, obligatoria, con 9 ECTS del primer curso del Grado en Ingeniería de Sistemas de Telecomunicación, Sonido e Imagen (GISTSI).

- Física Aplicada: asignatura anual, obligatoria, con 9 ECTS, del primer curso del GISTSI.

- Herramientas matemáticas aplicadas a las Telecomunicaciones: asignatura semestral, optativa, con 4'5 ECTS, del cuarto semestre del GISTSI.

- Instrumentos de Estadística y Simulación: semestral, obligatoria, con 6 ECTS, del tercer semestre del Grado en Ciencias Ambientales (GCCAA).

Posteriormente se ha ampliado a otras asignaturas como prácticas de Física del Grado de Diseño Industrial y Fundamentos Físicos de la Informática del Grado de Ingeniería 
Informática, del Campus de Alcoy y en Física del GCCAA. En este trabajo se detallan cuáles son los pasos que se están siguiendo en el desarrollo de este proyecto así como los materiales que se están elaborando.

Para aplicar el Flipped Teaching, se requieren varias etapas previas, importantes para el éxito posterior de la puesta en práctica metodológica: Seleccionar los contenidos adecuados para un aprendizaje autónomo; determinar la metodología Flip para dichos contenidos; buscar y seleccionar recursos y materiales desde el repositorio RiuNet de la UPV u otros; crear, en su caso, nuevos recursos, preferentemente objetos de aprendizaje para RiuNet; elaborar fichas de programación de clases Flip para cada una de las asignaturas implicadas en el proyecto que contienen los datos básicos de la asignatura, el tema o sección de aplicación, los objetivos de aprendizaje, el diseño de la actividad Flip asociada (tareas de aprendizaje para realizar en casa y actividades a realizar en clase), los recursos a utilizar por el alumnado durante las tareas de aprendizaje no presencial, enlaces y su descripción, otros posibles recursos y finalmente la evaluación de la actividad Flip. Las fichas pueden ser útiles para cualquier profesor con docencia similar a las asignaturas consideradas. También se han elaborado fichas de programación Flip mixtas, interdisciplinares, para la coordinación entre la asignatura de Matemáticas 2 y de Física, creándose asi sinergias entre ellas. Otro objetivo del proyecto es ensayar algunas de las fichas elaboradas y registrar los resultados obtenidos.

\section{Desarrollo de la innovación}

Son varias las actuaciones que se están desarrollando durante el curso 2015-2016:

- A medida que se ha impartido la docencia, se ha reflexionado sobre la posibilidad de aplicar el nuevo enfoque en determinadas secciones de nuestras unidades temáticas, generando las fichas Flip correspondientes. Para alguna de las fichas Flip se han tenido que generar vídeos de elaboración propia (Polimedias y screencast) debido a que la nomenclatura y el enfoque que se buscaba no era la que aparecía en vídeos de la red.

- Se están creando sinergias entre las asignaturas de Matemáticas y Física, dando lugar a la creación de fichas Flip matemáticas que serán utilizadas en Física.

- En algunos casos ya se ha puesto en marcha alguna de las actividades Flip propuestas en las fichas creadas, que pasamos a describir a continuación.

\subsection{Física del GISTSI}

Se ha aplicado la metodolgía Flip en los temas relativos a la Termodinámica: Temperatura y Calor, y a la segunda ley. La actividad Flip propuesta, voluntaria, y desarrollada en el primer semestre de este curso 2015-2016, contemplaba la realización de un PBL (Alba, del Rey, Vidal y Roig, 2015). Este tipo de estrategia puede ayudar a que los futuros ingenieros se acerquen a un proyecto real de ingeniería, antes de finalizar su titulación (Case y Light, 2011). Se asigna un PBL por defecto, pero los grupos podían elegir algún otro tema alternativo. La actividad se desarrolla durante 4 semanas y el resultado final es una exposición de todos los PBLs. La ficha Flip se expone en la Figura 4.

(cc) EY-NC-ND 2016, Universitat Politècnica de València

Congreso In-Red (2016) 


\begin{tabular}{|c|c|}
\hline $\begin{array}{l}\text { INNOVACION } \\
\text { UPV }\end{array}$ & $\begin{array}{c}\text { PROYECTO DE INNOVACIÓN Y MEIORA EDUCATIVA } \\
\text { CURSO 2015-2016 } \\
\text { Estudio sobre la aplicación del Flip Teaching en asignaturas de Matemáticas y Fisica }\end{array}$ \\
\hline \multicolumn{2}{|c|}{ 1. DATOS BASICOS DE LA ASICNATURA } \\
\hline \multicolumn{2}{|c|}{$\begin{array}{l}\text { Nombre: FISICA. Código: } 11264 \\
\text { Grado en Ingenieria de Sistemas de Telecomunicación, Sonido e Imagen. Carácter: Formación bäsica } \\
\text { Créditos: } 9,00 \text {-Téoria: } 4,5 \text {-Prácticas: } 4,5\end{array}$} \\
\hline \multicolumn{2}{|c|}{ 2. TEMA-SECCION } \\
\hline \multicolumn{2}{|c|}{$\begin{array}{l}\text { Temas } 6 \text { y 7. Termodinámica: Temperatura y Calor. La segunda tey. } \\
\text { PBLs sobre termodinámica }\end{array}$} \\
\hline
\end{tabular}

\section{OBJETIVOS DE APRENDIZAJE}

Al finalizar la actividad FLP propuesta, el alumnado será capaz de;

- Explicar las diferencias entre calor y temperatura

o Explicar las diferentes escalas termamétricas y tipos de termómetros

o Formular con expresiones matemáticas con los efectos del calor

- Identificar la primera Ley de la Termodinámica

o Formular la transmisión de energla a través de la conducción, convección y radiación.

- Explicar el concepto de máquina térmica

- Identificar los ciclos conocidos (Carnot y otto)

- Identificar y formular la segunda ley de la termodinámica

\section{ACTIVIDAD FLIP}

Realización de PBL.s de los dos temas. Se asigna un PBL por defecto pero los grupos pueden elegir temas. alternativos. La descripción del PBL básico es la siguiente:

TITULO DEL PROYECTO: Fabricación y calibración de un termómetro casero.

ENUNCIADO: Diseñar y fabricar un termómetro casero con cierta calibración. Debe permitir ciertos rangos de temperatura. Debe aportarse como minimo un poster en A3 (cambiable por video o similar).

PREGUNTA MOTRIZ ¿Todos los termámetros son de mercurio?

PLANIFICACIÓN: 4 semanas

Semana 1: Búsqueda de información y lectura de materiales.

Semana 2: Trabajo en grupo. Entregable 1: descripción del posible prototipo y materiales a utilizar. Previsión de cálculos teóricos del prototipo.

Semana 3: Trabajo en grupo. Avance de borrador de poster a similar. Problema de clase 3 y test. Semana 4: Presentación prototipos en clase

5. RECURSOS Y DESCRIPCION

En la descripción del Pal basico se recomiendan los siguientes videos de construcción de termómetros:

hittos:/ wmw youtubecom/watch?' $=2121291$ Sins

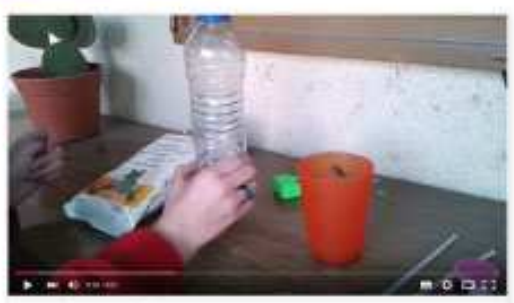

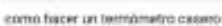

(cc) EY-NC-ND 2016, Universitat Politècnica de València

Congreso IN-RED (2016) 


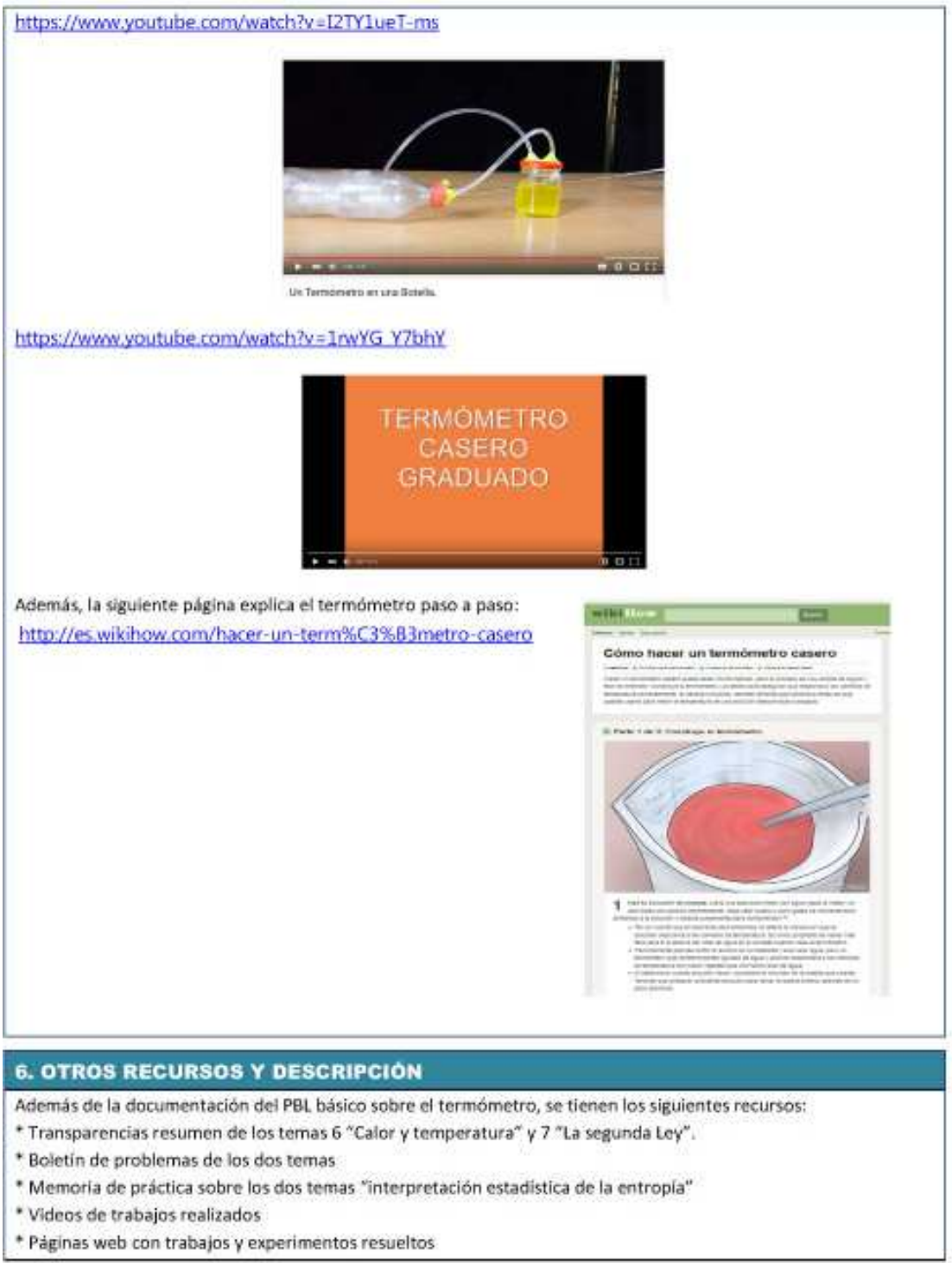

\section{EVALUACION}

Se valoran 20 puntos (sobre 400 puntos) en tres partes; el entregable 1 , el poster o similar y la presentación. Para la presentación se prepara rúbrica de profesores y alumnos.

Fig.4 Ficha Flip sobre Termodinámica

\subsection{Prácticas de Matemáticas 2 del GISTSI}

Se han invertido las clases de laboratorio informático de la asignatura Matemáticas 2 del GSITSI. Hasta este curso el alumno disponía de unos guiones de prácticas (en formato pdf) que eran explicados por el profesor en la sesión, siendo éste el que dirigía la práctica y exigía que los alumnos repitieran sus mismos pasos con el programa informático utilizado (en este

(c)) EY-NC-ND 2016, Universitat Politècnica de València

Congreso In-Red (2016) 
caso Matlab). El alumno se limitaba a repetir lo que indicaba el guión de prácticas. Los últimos minutos se dedicaban para explicar un trabajo, que en grupos de tres, debían realizar y entregar en un plazo de diez días. La nota de prácticas provenía de la corrección de este trabajo. Con la inversión de la clase, se pide a los alumnos que traigan el material trabajado, que en este caso no es un simple guión .pdf. Una de las herramientas disponibles para la creación de contenidos en la plataforma educativa PoliformaT de la UPV, son las Lessons. Con esta herramienta se ha reemplazado el guión pdf, permitiendo insertar vídeos, Polimedias y screencast (algunos creados específicamente), además de permitir enlazar con Exámenes y Tareas de PoliformaT. Al estudiante se le pedía que se preparase el Lesson correspondiente y la realización de un test en Exámenes de PoliformaT, con un plazo temporal que finalizaba el día previo a la sesión práctica. La sesión presencial (2 horas por práctica) se planificó de la siguiente forma:

- Ofrecer feedback: primero respecto a las dudas del alumnado en cuanto al contenido del Lesson y en segundo lugar respecto a las preguntas del test realizado.

- Explicación del trabajo a realizar en grupo: a partir de un documento disponible en Tareas de PoliformaT.

- Realización del trabajo propuesto por parte de los estudiantes.

Al finalizar la práctica los estudiantes depositan en la Tarea correspondiente la resolución del trabajo.

Desde la primera práctica, aquellos alumnos que no realizaron el test, y que no se habían preparado el Lesson correspondiente, se les ubica en la última fila del aula, separados del resto de estudiantes. Su trabajo durante la sesión de prácticas consistía en realizar la tarea de casa (preparación del Lesson) y realización del test, debiendo obtener al menos un 6 en dicho test para poder realizar el trabajo en grupo y así obtener la puntuación de la práctica. Se les daba un día de plazo para la realización de dicho trabajo. En general ninguno de estos alumnos entregó el trabajo, pero la experiencia sirvió de motivación para que en las siguientes prácticas se acoplaran a la nueva metodología.

En la Figura 5 presentamos parte de la ficha Flip correspondiente a la tercera de las prácticas.

\subsection{Instrumentos de Estadística y Simulación del GCCAA}

Se aplicó en las clases de inicio de la asignatura para repasar conceptos previos, aunque los resultados no fueron muy alentadores, por la falta de seguimiento de los estudiantes en cuanto a la parte de aprendizaje autónomo. Esta experiencia refuerza la idea de exigir la realización de un test después del autoaprendizaje. Esta experiencia previa se ha tenido en cuenta al elaborar las fichas Flip correspondientes, una de las cuales se ilustra en la Figura 6.

(cc) BY-NC-ND 2016, Universitat Politècnica de València

Congreso IN-RED (2016) 


\begin{tabular}{|c|c|c|}
\hline $\begin{array}{l}\text { INNOVACION } \\
\text { UPV }\end{array}$ & \multicolumn{2}{|c|}{$\begin{array}{l}\text { PROYECTO DE INNOVACIÓN Y MEIORA EDUCATIVA } \\
\text { CURSO 2015-2016 } \\
\text { Estudio sobre la aplicación del Flip Teaching en asignaturas de Matemáticas y Fisica }\end{array}$} \\
\hline \multicolumn{3}{|c|}{ 1. DATOS BASICOS DE LA ASIGNATURA } \\
\hline \multicolumn{3}{|c|}{$\begin{array}{l}\text { Nombre: Matemáticas 2. Código: } 11265 \\
\text { Grado en Ingenieria de Sistemas de Telecomunicación, Sonido e Imagen. Carácter: Formación básica } \\
\text { Créditos: 9,00 -Teoria: 5,15 -.Prácticas: } 3,85\end{array}$} \\
\hline \multicolumn{3}{|c|}{ 2. TEMA-SECCION } \\
\hline \multicolumn{3}{|c|}{ Bloque V: Fundamentos matemáticos con Matlab. Prácticas informáticas: Ajuste e interpolación } \\
\hline \multicolumn{3}{|c|}{ 3. OBJETIVOS DE APRENDIZAJE } \\
\hline \multicolumn{3}{|c|}{$\begin{array}{l}\text { Al finalizar la actividad Flip propuesta, el alumnado será capaz de: } \\
\text { I Ientificar qué tipo de ajuste se corresponde a una colección o nube de puntos } \\
\text { - Calcular y representar con Matlab el polinomio que mejor ajusta a una nube de puntos } \\
\text { Diseñar trayectorias polinómicas que pasen por un conjunto de puntos ( } x, y \text { ). } \\
\text { - Aproximar funciones no polinómicas con polinomios. } \\
\text { - Diseñar trayectorias con funciones polinómicas a troz.os (splines) a partir de una tabla de datos }\end{array}$} \\
\hline \multicolumn{3}{|c|}{ 4. ACTIVIDAD FLIP } \\
\hline \multicolumn{3}{|c|}{$\begin{array}{l}\text { Realización de una unidad de Lessons: } \\
\text { Matlab 3: Ajuste e interpolación. } \\
\text { 1. El alumnado debe, previa a la realización de la sesión de prácticas, hacer un estudio de esta unidad. } \\
\text { 2. Una vez estudiada debe realizar un examen tipo test de Poliformat hasta el dia antes de la realización } \\
\text { de la práctica. } \\
\text { 3. En la sesión de la práctica el alumno podrá preguntar dudas pendientes. Posteriormente el profesor } \\
\text { comenta los resultados del test y realiza un feedback. } \\
\text { 4. Realización de un trabajo en grupo de dos alumnos, consistente en la resolución de dos problemas: } \\
\text { Utilizar un ajuste lineal para comparar la calidad acústica de un mismo recinto acústico con dos } \\
\text { tipos distintos de acondicionamiento, teniendo en cuenta la homogeneidad de los niveles de } \\
\text { presión sonora. } \\
\text { Modelizar una ruta o camino de wikiloc con la interpolación segmentaria con splines cúbicos. }\end{array}$} \\
\hline \multicolumn{3}{|c|}{ 5. RECURSOS Y DESCRIPCIÓN } \\
\hline \multicolumn{3}{|c|}{$\begin{array}{l}\text { 1. Una introducción al ajuste de datos con Matlab } \\
\text { https://media.upv.es/plaver/?autoplay=true\&id=6a490410-1760-6142-bab7-1931cb46d39f }\end{array}$} \\
\hline & 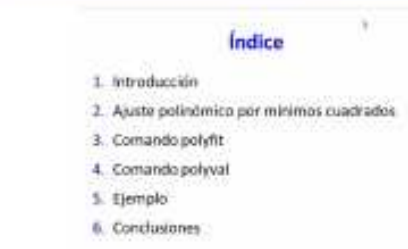 & $x^{2}=$ \\
\hline
\end{tabular}

\section{EVALUACIÓN}

El examen tipo test previo a la realización de la práctica se tiene en cuenta para poder realizar el trabajo en grupo. El trabajo en grupo realizado durante la sesión de prácticas se valora con un 0,2 sobre la nota del acta.

Fig.5 Parte de la ficha Flip sobre ajuste e interpolación 


\begin{tabular}{|c|c|}
\hline $\begin{array}{c}\text { INNOVACION } \\
\text { UPV }\end{array}$ & $\begin{array}{l}\text { PROYECTO DE INNOVACIÓN Y MEJORA EDUCATIVA } \\
\text { CURSO 2015-2016 } \\
\text { Estudio sobre la aplicación del Flip Teaching } \\
\text { en asignaturas de Matemáticas y Fisica }\end{array}$ \\
\hline
\end{tabular}

1. DATOS BÁSICOS DE LA ASIGNATURA

Nombre: Instrumentos de estadistica y simulación. Código: 10089

139-Grado en Ciencias Ambientales. Carácter; Obligatorio

Créditos: 6,00-Teoria: 4,00 --Prácticas: 2,00

\section{TEMA-SECCION}

Unidad Temática 1. Estadistica Descriptiva Univariante. Sección 1.1 Tablas de distribución de frecuencias

\section{OBJETIVO DE APRENDIZAJE}

Al finalizar la actividad Flip propuesta, el alumno será capaz de:

- Elaborar una tabla de distribución de frecuencias a partir de un conjunto de datos: cualitativos, cuantitativos discretos o cuantitativos continuos.

- Elaborar los principales gráficos asociados a la información de las tablas de distribución de frecuencias.

- Interpretar la información que proporciona cualquier tabla de distribución de frecuencias.

- Interpretar la información que proporciona cualquier gráfico asociado a una tabla de distribución de frecuencias

\section{ACTIVIDAD FLIP}

4.1 Lectura del tema 1: Tablas de distribución de frecuencias y representaciones gráficas (manual de apuntes). [Tiempo estimado $45^{\prime}$ ].

4.2 Realización de un test Test via Poliformat [Tiempo estimado 15'].

4.3 Los que no obtengan un $75 \%$ de aciertos en el test, deben volver a leer el tema y/o apoyarse en los videos y realizar otra vez el test. (Tiempo estimado $60^{\prime}$ ).

4.4 En clase se realizaran problemas del tema 1, dirigidos a que adquieran la competencia de saber construir tablas de frecuencias y gráficos, e interpretarlos. (Tiempo estimado $90^{\prime}$ ).

\section{RECURSOS Y DESCRIPCIÓN}

- Apuntes del tema 1: Tablas de distribución de frecuencias y representaciones gráficas. Se trata de ficheros en formato pdf disponibles en Recursos de PoliformaT.

- https://www.youtube.com/watch?v=OzS7xkOUaE0

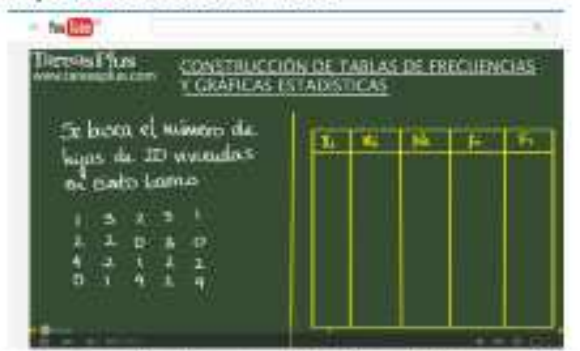

Video donde se describe la construcción de una de tablas de frecuencias para datos discretos, y la posterior definición de las gráficas estadisticas: de barras, circular y poligono de frecuencias. 
- https://www.youtube.com/watch?v=kB7iDbOsbpl

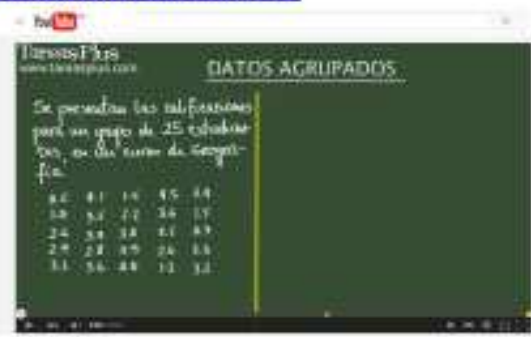

Video donde se describe la construcción de una de tablas de frecuencias para datos continuos

2. https://media.upv.es/plaver/?id=fdged949-291f-05-43-b843-46ch45025d87

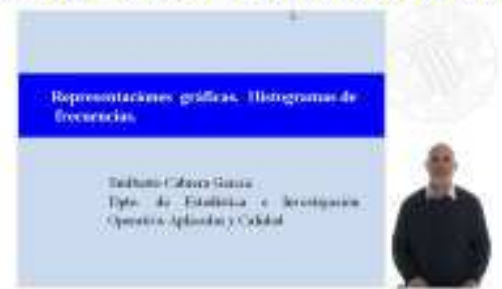

Video Polimedia de Suiberto Cabrera que trata sobre la construcción de Histogramas y su interpretación

\section{OTROS RECURSOS Y DESCRIPCION}

○ https://canal.uned.es/mmobj/index/id/10703.html

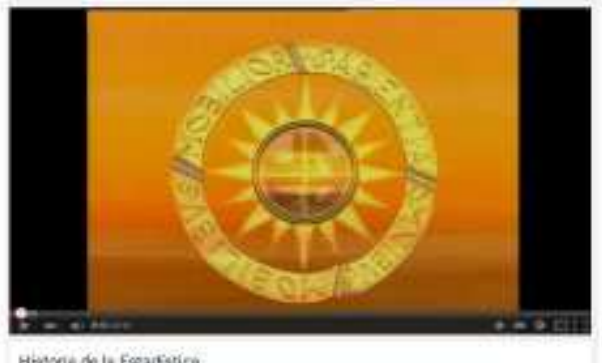

Video de la UNED, de la serie La Aventura del Saber, dedicado a la Historia de la Estadistica.

\section{EVALUACIÓN}

No se realizará un acto de evaluación particular para la secciỏn descrita en esta ficha.

Se realizará de un acto de evaluación global una vez completada la Unidad Temática 1 (Estadística Descriptiva Univariante) para valorar la adquisición de las competencias correspondientes a toda la unidad. Sölo tendrian derecho los que hayan realizado positivamente los test y asistido a las clases (Tiempo estimado $120^{\prime}$ ).

Su peso en la evaluación final será de 1 punto

Fig. 6 Ficha Flip sobre tablas de distribución de frecuencias

(cc)) EY-NC-ND 2016, Universitat Politècnica de València

Congreso In-Red (2016) 


\section{Resultados}

Las fichas Flip generadas hasta el momento son las siguientes:

Física: campo magnético, campo eléctrico, termodinámica, modelización e incertidumbre en la medida.

Matemáticas: tablas de distribución de frecuencias, medidas de tendencia central, medidas de dispersión y de forma, curvas en forma paramétrica y polar, ajuste e interpolación, introducción a las series numéricas.

Interdisciplinares (Física y Matemáticas): producto escalar, producto vectorial, propagación de errores y repaso de derivadas de funciones de una variable y su extensión a funciones de varias variables.

Los resultados correspondientes a las prácticas del primer semestre de Matemáticas 2 han mejorado respecto a las del curso anterior, como muestra la Figura 7.

\begin{tabular}{|c|c|c|}
\cline { 2 - 3 } \multicolumn{1}{c|}{} & $\begin{array}{c}\text { \% estudiantes } \\
\text { con nota } \geq \mathbf{5}\end{array}$ & $\begin{array}{c}\text { Nota media entre los } \\
\text { estudiantes con nota } \geq \mathbf{5}\end{array}$ \\
\hline $\mathbf{2 0 1 4 - 2 0 1 5}$ & 64.3 & 7.8 \\
\hline $\mathbf{2 0 1 5 - 2 0 1 6}$ & 76.8 & 8.6 \\
\hline
\end{tabular}

Respecto a los PBL de la asignatura de Física del GISTSI, el número de participantes ha sido de 33 sobre 53 (era voluntario), en equipos de 3 o 4 alumnos. Respecto a las notas, todas entre 16 y 20 puntos sobre 20, de un total de 400 puntos de la evaluación de la asignatura. Los proyectos que presentaron versaban sobre los siguientes temas: termómetros de gas, prototipo de barco a vapor, coches a vapor, drinking bird y una central termoeléctrica.

\section{Conclusiones}

El grupo GIERMAC está desarrollando el proyecto "Estudio sobre la aplicación del Flip Teaching en asignaturas de Matemáticas y Física" dentro de la convocatoria PIMEs 20152016 de la UPV. En este trabajo se han detallado los pasos seguidos en el desarrollo de este proyecto y se han expuesto muestras de los materiales creados (fichas Flip). Durante el primer semestre del curso 2015-2016 ya se han puesto en marcha algunas actividades utilizando el enfoque expuesto de la la metodología Flip, obteniendo, sobre todo en las asignaturas de Física Aplicada y Matemáticas 2 del GISTSI, buenos resultados.

\section{Agradecimientos}

Este trabajo cuenta con el apoyo de la convocatoria PIMEs 2015-2016, convocada por el Vicerrectorado de Estudios, Calidad y Acreditación de la UPV. 


\section{Referencias}

ALBA, J., DEL REY, R., VIDAL, A. Y ROIG, B. (2015) "Aprendizaje Basado en Proyectos en el Grado en Ingeniería de Sistemas de Telecomunicaciones, Sonido e Imagen. Caso práctico del electroscopio como experiencia interdisciplinar entre Física y Matemáticas" en 23 CUIEET. Valencia. Escuela Técnica Superior de Ingeniería del Diseño. 1-11.

BERGMANN, J. Y SAMS, A. (2015). Dale la vuelta a tu clase: Lleva tu clase a cada estudiante, en cualquier momento y cualquier lugar. SM.

CASE, J. M. Y LIGHT, G. (2011). "Emerging Methodologies in Engineering Education Research" en Journal of Engineering Education, vol. 100, issue 1, p. 186-210

GANNOD, G., BURGE, J. Y HELMICK, M. (2008). "Using the inverted classroom to teachsoftware engineering" en Proceedings of the International Conference on Software Engineering (ICSE). Leipzig, Germany. p. 10-18

HUGHES, H. (2012). "Introduction to Flipping the College Classroom" en Proceedings of world conference on educational multimedia, hypermedia and telecommunications, Chesapeake, p. 2434-2438

LAGE, M.J., PLATT, G.J. Y TREGLIA, M. (2000). "Inverting the classroom: A gateway to creating an inclusive learning environment" en The Journal of Economic Education, vol. 31, issue 1, p. $30-43$

LÓPEZ MORENO, M. (2015). “¿Qué es el aula invertida?” en Nubemia, tu academia en la nube, enero de 2015. < http://www.nubemia.com/aula-invertida-otra-forma-de-aprender/> [Consulta: $21 \mathrm{de}$ marzo 2016]

MORAVECD, M., WILLIAMS, A., AGUILAR-ROCA, N. Y O'DOWD, D.K. (2010). "Learn before lecture: a strategy that improves learning outcomes in a large introductory biology clas" en $C B E$ Life Sci Educ, vol. 9, p. 473-481

PALMER, T. Flipping Physic. < http://www.flippingphysics.com/flipping.html> [Consulta: 21 de marzo de 2016]

SANTIAGO, R. Y DIEZ, A. The flipped classroom. < http://www.theflippedclassroom.es/>[Consulta: 21 de marzo de 2016]

TALBERT, R. (2012). "Learning MATLAB in the Inverted classroom" en Proceedings of the ASEE Annual Conference. San Antonio, Texas. 25.883.1-25.883

TALBERT, R. (2012). “Inverted Classroom” en Colleagues, vol. 9, issue 1, article 7

TALBERT, R. (2014). "Inverting the Linear Algebra Classroom" en PRIMUS (Problems, Resources, and Issues in Mathematics Undergraduate Studies), vol. 24, issue 5, p. 361-374

THOMAS-PALMER, J. "Showing the Differences between a Traditional and a Flipped Classroom (6:52)"< http://www.flippingphysics.com/flipping.html > [Consulta: 21 de marzo de 2016]

TOURÓN, J., SANTIAGO, R. Y DIEZ, A. (2014). The Flipped Classroom: Cómo convertir la escuela en un espacio de aprendizaje. Lugar de publicación: Grupo Océano. Digital-Text

TOURÓN, J. Javier Touron. Talento-Educación-Tecnología. http://www.javiertouron.es/> [Consulta: 21 de marzo de 2016]

(cc) EY-NC-ND 2016, Universitat Politècnica de València

Congreso In-Red (2016) 